\title{
Differentiate: A Concept Analysis
}

Katie Bonneau ${ }^{1}$, Courtney Neurohr ${ }^{2}$ and Sharon L. Van Sell ${ }^{3 *}$

${ }^{1}$ Graduate Nursing Program, Texas Woman's University, United States of America

${ }^{2}$ Graduate Nursing Program, Texas Woman's University, United States of America

${ }^{3}$ Professor, The Houston J. and Florence A. Doswell College of Nursing, Texas Woman's University, United States of America

\section{Abstract}

The authors developed the concept differentiate as applied to the advanced practice nurse (APN) due to the lack of a current theory regarding the subject in the existing nursing literature. For instance, the APN uses differential diagnosis daily in practice. A concept analysis of differentiate according to the Walker and Avant methodology and applied to the APN can assist the APN to conduct a differential diagnosis accurately to create a safer environment for the patient. Attributes of the concept differentiate, include the following: (a) critical thinking, (b) evidence-based literature, (c) Distinguish, (d) differences, (e) complexity, and (f) complete. In addition to attributes of the differentiate concept, antecedents and consequences further elucidate the concept differentiate, and a model representation combines these three aspects to create a complete picture of the concept differentiate applied to the APN. A model, borderline, and contrary case provide real-life APN examples of the concept. Finally, the authors explore the method for conducting a proper differential diagnosis in the APN's practice to begin the patient's healing process.

\section{Publication History:}

Received: April 04, 2018

Accepted: June 21, 2018

Published: June 23, 2018

\section{Keywords:}

Advanced practice nurse, Differential diagnosis, Differentiate, Nursing, theory
Over time, advanced practices nurses (APNs) have used differential diagnoses daily to delineate probable diagnoses for patients. Choosing the proper diagnosis most often resulted in proper treatment of the patient and has led to a faster healing process. Applying Walker and Avant's [1] eight-step process to understand the concept differentiate applied to the APN, the authors were able to make a clear distinction between defining characteristics and unrelated concepts. The purpose of the concept analysis was to enable APNs to understand the critical attributes of the concept differentiate to conduct a proper differential diagnosis. The attributes associated with the concept differentiate included the following: (a) critical thinking, (b) evidence-based literature, (c) distinguish, (d) differences, (e) complexity, and (f) complete.

The concept analysis involved a complex process that forced the APN to use the proper tools to differentiate among various disease processes. To conduct a differential diagnosis properly, an APN must first understand the realm of an APN. The five domains of advanced practice nursing included "autonomy, critical thinking, decision making, values-based care and improving practice" [2]. To afford high-quality care and apply all five domains, an APN must have acquired effective cognitive skills that allow him or her to assess the patient's presenting problem critically. The accurate assessment consisted of knowing the appropriate questions to ask during a detailed history and understanding the correct techniques to employ during a thorough physical assessment. For example, how should the APN begin to assess a patient who presents with abdominal pain? Due to the vast amount of diagnoses associated with the complaint of abdominal pain, the APN must be able to recognize patients with lifethreatening conditions and identify high-risk populations frequently associated with differential diagnoses such as peritonitis, perforation, or obstruction [3]. A detailed history and focused assessment enabled the APN to triage a patient properly.

Following the assessment, the APN utilized her autonomy to order the correct laboratory and other tests to begin narrowing down diagnoses and to determine the acuity of the patient's condition. Values-based care incorporated into ordering the right tests. In addition, APNs should always keep the patient in mind, know the patient's preferences before caring for him or her, and understand the patient's limits. APNs must also have a solid foundation for evidencebased practice to understand the suitable labs and tests to run. For example, if a patient were to present to the office with frequent stomach aches and irritation to the bowel, the APN must not quickly jump to the conclusion that the patient has irritable bowel syndrome that requires lifestyle changes, medication management, and other costly efforts. After conducting a detailed history, including foods that upset the patient's stomach, the APN should use current evidence to conduct proper tests, including a lactose hydrogen breath test. If the patient instead has lactose intolerance, the APN saved the patient time, resources, and a multitude of lifestyle changes.

Finally, the reflection deemed as a crucial step for an APN who strives to improve practice. On a daily basis, APNs make mistakes. The APN should use mistakes to educate himself and to enable the entire healthcare team to grow and finesse clinical practice skills. The APN must ask himself what steps were missed to reevaluate how to improve conducting a differential diagnosis.

The ultimate goal of understanding the concept of differentiate was for the APN to avoid misdiagnosis and for the patient to heal promptly. The concept of differentiate related to the APN was important to analyze because understanding the attributes of a differential diagnosis may provide a safer utilization of practice for an $\mathrm{APN}$ and a more secure environment for the patient.

"Corresponding Author: Dr. Sharon L. Van Sell, The Houston J. and Florence A Doswell College of Nursing, 5500 Southwestern Medical Avenue, \#7209, Dallas, Texas, USA, 75235-7299. Tel: 1-864-275-3527, Fax: 214-689-6539; E-mail: svansell@twu.edu

Citation: Bonneau K, Neurohr C, Van Sell SL (2018) Differentiate: A Concept Analysis. Int J Nurs Clin Pract 5: 280. doi: https://doi.org/10.15344/2394$4978 / 2018 / 280$

Copyright: (c) 2018 Van Sell et al. This is an open-access article distributed under the terms of the Creative Commons Attribution License, which permits unrestricted use, distribution, and reproduction in any medium, provided the original author and source are credited. 


\section{Concept Analysis}

\section{Aim of the concept analysis}

The purpose of conducting concept analysis on the concept of differentiate was to prevent misdiagnosis and to understand better a differential diagnosis applied to the APN, providing a more secure healing environment for the patient. Currently, no formed theories of the concept differentiate applied to the APN were available, even though APNs used differential diagnosis every day. According to Walker and Avant [1], the aim of a concept analysis was to clarify ambiguous concepts and give the concept specific meaning to explain the desired results. Through the process of differential diagnosis, APNs determined how quickly to treat the patient. APNs needed to distinguish between possible causes of the disease process by running certain laboratory and other tests to narrow down the possible diagnoses. Once correctly diagnosed and with proper medical treatment, the enabled patient begins the healing process. The purpose of the concept analysis assisted APNs in understanding the critical attributes of the concept differentiate to conduct an appropriate differential diagnosis.

A concept analysis of the concept differentiates applied to the APN encouraged student and new APNs to learn the framework of a differential diagnosis and to apply the learned material in the health care field. Philosopher John Dewey [4] believed that students learned educational material best when they incorporated their learning with hands-on experience. A mentor who guided the student or new APN through the systematic steps of a differential diagnosis further enhanced his understanding. As a result, the APN gained a richer understanding of the differential diagnosis process.

According to Scordo [5], "Whereas non-analytic reasoning is subconscious, analytic or diagnostic reasoning is a conscious, active, and analytic process". Nonanalytic reasoning occurred as a result of an abundance of experience from past patient situations. When a practitioner subconsciously recalled past medical patient scenarios, he utilized past patient information when assessing a current patient with similar problems. The hallmark of an individual's subconscious knowledge or tacit knowledge experienced, and experience enabled pattern recognition [5]. Employing past cases with similar presentations enabled APNs to assess a patient more quickly and effectively, conduct a differential diagnosis, and produce an accurate diagnosis with an appropriate treatment plan that also coincides with the patient's values. Although tacit knowledge required expertise, the APN understood that the use of nonanalytic reasoning alone could lead to possible errors when diagnosing a patient.

Alternatively, analytic reasoning included deductive reasoning and inductive reasoning. Deductive reasoning began with general information and moved to more specific information; in contrast, inductive reasoning started with specific information and moved to broader information. APNs used deductive reasoning to conduct a full assessment, ask a series of questions while actively listening to patient complaints, and collect data [5]. Many practitioners may have wondered how to consider all the possible hypotheses and information before forming a diagnosis [6]; however, listing every potential cause of the disease process was impossible, especially under time constraints. Consequently, APNs needed to ask pertinent questions and form multiple differential diagnoses, including the highest risk diagnoses, early in the conversation [6]. The data either agreed with or denied particular diagnoses, prompting the APN to ask more specific questions to drive data collection. As APNs grew into their role, they were able to point out key findings that accelerated the differential diagnosis process [5].

Inductive reasoning was another analytic strategy requiring the APN to decide which objective signs and symptoms were pertinent to the correct diagnosis. Using information from a detailed history and physical guided the path to a proper diagnosis. APNs employed a cognitive algorithm process that reduced the number of potential diagnoses [5]. Although inductive and deductive reasoning comprised two probable paths for conducting a differential diagnosis, the more popular type of analytic reasoning strategy has remained deductive reasoning.

APNs incorporated their understanding of the concept differentiate into practice by completing a thorough assessment of patients to create a complete picture of each patient and his or her symptoms. APNs must grasp the entire clinical picture of the patient to help determine a correct diagnosis. By diagnosing patients properly and promptly, APNs enabled their patients to regain a state of wellbeing sooner. A better understanding of the concept differentiates helped to prevent misdiagnosis and to accelerate a differential diagnosis from the APN, creating a secure healing environment for the patient.

\section{Relating Theory to Practice}

The defined concept differentiate enabled the scholarly APN community to understand the key characteristics, antecedents, and consequences of a differential diagnosis as applied to the APN. Thorough and clear understanding of the concept differentiates decreased ambiguity and enabled future APNs to test the concept in practice. To date, no identified theories were available that related the concept differentiate to the APN; however, Meleis [7] incorporated the concept differentiate when she proposed three strategies to develop conceptual meaning regarding nursing theory, research, and practice, including concept exploration, concept clarification, and concept analysis. Meleis defined differentiating to mean sorting similarities and differences between the developed concept and other related concepts; therefore, the development of the concept differentiate applied to the APN in practice was necessary to improve the APN's understanding of conducting a thorough and complete differential diagnosis.

\section{Definitions and Uses of Differentiate}

In the field of healthcare, medical doctors traditionally have used the concept differentiate as a vital process to diagnose patients. The complex, systematic process involved evidence-based knowledge and critical thinking to narrow down potential causes of a disease process ultimately to delineate a proper diagnosis. In recent years, APNs have come into demand, creating a need for the general public as well as APNs to understand the role of the APN. Although physicians have in the past undertaken the responsibility of conducting a differential diagnosis, the newer designation of APN has also utilized the differential diagnosis process. Consequently, a better understanding of the APN's thought process to differentiate one diagnosis from another became necessary. Based on a thorough literature review of the concept differentiate applied to the APN, no current theories were evident regarding differentiating regarding the APN or differential diagnosis in relation to the APN. 
The concept differentiat varied based on the context, such as education, healthcare, or mathematics. The Merriam-Webster Online Dictionary [8] defined differentiate as to "constitute a difference that distinguishes", "to develop differential characteristics in", "to cause differentiation of in the course of development", and "to express the specific distinguishing quality of: discriminate". The definition of the word differentiates accurately provided key characteristics for applying the concept differentiate to the APN.

A student APN and a new APN better understand how to differentiate by researching the current literature that reviewed a certain disease process. Furthermore, the student APN and new APN need to learn to delineate similar health conditions by conducting a focused assessment and proper diagnostic testing. No current theory regarding differentiation and the APN emerged. Conducted a literature search by using a combination of the words "differentiate," "differential diagnosis," "advanced practice nurse," and "nurse practitioner" to help clarify antecedents, attributes, and consequences of the concept differentiate applied to the nurse practitioner. An electronic database search included Medline with Full Text (EBSCO) and CINAHL Complete.

Extracted from the current literature, the defining attributes of the concept differentiate applied to the APN were: (a) critical thinking, (b) evidence-based literature, (c) distinguish, (d) differences, (e) complexity, and (f) complete. The antecedents preceded the attributes of the concept differentiate in the context of the APN, and the consequences resulted from the attributes. According to Walker and Avant [1], attributes of the concept were the consistently defined characteristics of the concept, and the attributes needed to exist to provide a broad insight into the defined concept.

Based on the literature search, all scholarly authors agreed that the evidence-based literature characterized the concept differentiate applied to the APN. Most authors explained how APNs assessed the differences between signs and symptoms of various diagnoses when performing a differential diagnosis [3,5,9-13]. APNs compared and contrasted differences of the possible diagnoses to distinguish among the identified diagnoses. The APNs discriminated among the differences in potential diagnoses, a skill that took time to improve, involved critical thinking, and led to an accurate diagnosis [2,5]. An APN accurately conducted a differential diagnosis by sorting through the complexity of each potential diagnosis process $[2,5,12]$. Finally, all authors agreed that an APN differentiated among potential diagnoses by understanding the antecedents of a differential diagnosis, including a complete clinical picture of the patient's presenting history as well as knowledge of the pertinent signs and symptoms of the presenting illness.

The desired outcome of understanding the concept differentiate applied to the APN enabled the APN to differentiate signs and symptoms to diagnose his or her patient correctly to improve the patient's health. According to Walker and Avant [1], middle-range theories "contain a limited numbers of variables and are limited in scope". The group narrowed the literature search of the concept differentiate by including articles based solely on the APN and specifying the concept differentiate applied to the APN. Upon conducting research, the authors found many medical-related articles available online because physicians traditionally have used the differential diagnosis process to create a plan of care for patients. As a result, a concept analysis of the concept differentiate applied to the APN became helpful for APNs to understand the theoretical framework associated with a differential diagnosis; however, various medical domains were also able to utilize the concept analysis.

\section{Antecedents and Consequences}

Antecedents of the concept enhanced the defining characteristics of differentiate within a context. According to Walker and Avant [1], antecedents consisted of "events or incidents that must occur or be in place prior to the occurrence of the concept" (p. 167). The antecedents of the concept differentiate applied to the APN either enhanced or detracted from the defining attributes. For instance, autonomy was an antecedent that empowered the APN to believe that he or she was able to diagnose a patient correctly. In the concept analysis of differentiate applied to the APN, all authors agreed that a complete history and physical, review of systems, knowledge base, and expertise contributed to a more thorough differential diagnosis. In addition, a proper analysis through cognitive processes enhanced a differential diagnosis [5].

According to Walker and Avant [1], consequences of the concept analysis constituted the "events or incidents that occur as a result of the occurrence of the concept". Clinical outcomes were either positive or negative. Positive consequences of the concept differentiate included an accurate diagnosis, a path to wellness, and a refinement of skills; in contrast, a negative consequence may have been a misdiagnosis and subsequent illness of the patient, depending on the thoroughness of the antecedents and the defining attributes. Based on the primary diagnosis, the APN either aided the patient by prescribing a correct treatment course or potentially harmed the patient by issuing an incorrect treatment course. Regardless of the outcome, APNs should always reflect on the case to learn and grow in their role.

\section{Model Case}

A model case illustrated all of the defining attributes of the concept [1]. The following example demonstrated the key characteristics of the concept differentiate applied to the APN, including (a) critical thinking, (b) evidence-based literature, (c) distinguish, (d) differences, (e) complexity, and (f) complete.

A husband brought his wife into the emergency department because she has been complaining of lower abdominal pain that used to be dull and intermittent, but the pain has recently become sharp, continuous, and unbearable. Upon arrival to the hospital, the woman became unconscious. Due to the wide array of potential complex diagnoses, the APN critically thought and quickly acquired the patient's epidemiological factors, including gender, age of 30 years, and race as a White Caucasian to distinguish certain diagnoses regarding abdominal pain from others. Because of the patient's gender and childbearing age, the APN remembered that the current evidencebased literature said to perform an hCG test to determine pregnancy.

The APN then asked the patient's husband what other presenting symptoms his wife had. He said that his wife had mentioned that her period was more frequent with a brighter colored blood than usual. $\mathrm{He}$ had also observed his wife's becoming more nauseous with occasional vomiting episodes. These contextual clues were vital and allowed the APN to cluster the symptoms: The APN learned the character, location, and quality of the pain as well as associated symptoms. The APN next inquired about the wife's medical history, and the husband 
recalled that his wife had had only a tonsillectomy when she was a child. The APN then asked questions regarding the possibility of excessive drinking or psychiatric issues to infer if the patient hadrisk behaviors. To these questions, the husband answered "no."

Because of the patient's unstable state, time was of the essence. The APN performed an abdominal examination, starting with percussion followed by palpation while assessing the patient's reaction and body posture. The patient whimpered upon palpation in the lower abdominal region, but not in the upper region. The combination of a detailed history of subjective data with an objective assessment of the body allowed for a complete clinical understanding and led the APN to suspect ectopic pregnancy or ovarian torsion. Because the pain had persisted for some time and increased in severity, the APN determined that the diagnosis was likely ectopic pregnancy. The hCG test result showed greater than 2,000 IU/L, indicating pregnancy and confirming a diagnosis of ectopic pregnancy [3]. The APN immediately notified the surgeon to try to preserve future fertility and started an IV with packed red blood cells and fluid to resuscitate the patient from hypovolemic shock and possible mortality.

\section{Borderline Case}

A borderline case exemplified a scenario that contained some of the defining attributes of the concept [1]. For example, Carter and Attel [9] described differentiating between Crohn's disease and lactose intolerance. Carter and Attel accurately explained the differences between the food allergy lactose intolerance, food intolerance with irritable bowel syndrome (IBS), and the frequency of misdiagnosis between the two. However, Carter and Attel did not explore other diagnoses that might also display similar symptoms of diarrhea, abdominal pain, or nausea, such as colon cancer or a hernia. What was effective in the article by Carter and Attel was their use of current evidence-based literature to review the differing pathophysiology, tests, and treatment methods of lactose intolerance and IBS Furthermore, the authors used critical thinking to distinguish between the similarities and differences of the two mentioned diagnoses; however, due to the lack of a detailed history and physical, Carter and Attel presented an incomplete list of potential differential diagnoses that lacked a complex analysis associated with abdominal issues.

\section{Contrary Case}

A contrary case delineated an example that did not appropriately define the concept to contrast the defined attributes of the concept differentiate [1]. For example, Sarah was a new APN and felt overwhelmed by the number of patients she had to see in the clinic during the flu season. She walked into her first patient's room and quickly ran through her patient's signs and symptoms. Sarah hurriedly diagnosed the patient with the flu and prescribed Tamiflu for the patient. Later on that day, the clinic received a call from the same patient's husband, who reported that his wife was transported to an emergency department (ED) due to a continued decline in her health. After a thorough assessment of the patient, the ED then diagnosed the patient with toxic shock syndrome. This diagnosis was missed by the APN because she failed to complete a detailed assessment, and the APN did not ask the patient proper questions. An understanding of the concept of differentiated as it applied to the APN would have assisted the APN in the process of differentiating between disease processes to prevent mistakes that led to poor outcomes for the patient. By diagnosing properly, the APN could have treated the patient in a more timely manner, enabling a healing environment.

\section{Empirical Referents}

Empirical referents, according to Walker and Avant [1], were "classes or categories of actual phenomena that by their existence or presence demonstrate the occurrence of the concept itself. . . . They are means by which you can recognize or measure the defining characteristics or attributes". A defining attribute of the concept differentiate was critical thinking, and an empirical referent was a cross-sectional study by Lang, Beach, Patrician, and Martin [14]. This study by Lang et al. investigated 356 nurses of different experience levels and tested when nurses gained the skill of critical thinking [14]. The authors refuted the belief that years of experience had the biggest influence on critical thinking; however, neither age, level of education, nor employment type determined critical thinking test scores. Lang et al. suggested further research regarding how nurses use "clinical reasoning, intuition, and creativity in the clinical decision-making process" [14].

Evidence-based practice was an attribute of the concept differentiate that was also an empirical referent. In a study by Boström, Rudman, Ehrenberg, Gustavsson, and Wallin, et al. [15], the researchers conducted a cross-sectional study with 1,256 registered nurses and investigated the association among 18 independent variables and six evidence-based practices. Boström et al. concluded that the evidencebased practices required distinct cognitive skills, critical thinking, and reflection [15]. The authors also believed both the individual practicing and the organization's responsibility was to focus on the use of evidence-based practice to enhance healthcare.

\section{Impact of the Concept Differentiate}

Conducting a differential diagnosis involves a complex process. To distinguish among different disease processes, the APN must first understand the realm in which an APN belongs. The five domains of advanced practice include "autonomy, critical thinking, decision making, values-based care and improving practice" (p. 26) [2]. An APN must, therefore, have acquired effective cognitive skills that enabled him or she to assess the patient's presenting problem critically, including knowing the proper questions to ask during a detailed history and understanding the correct techniques to use during a thorough physical assessment. Once the APN has collected pertinent information, he or she must utilize autonomy to order the appropriate laboratory and other tests to begin narrowing down the possible diagnoses.

Incorporated into ordering the right tests was values-based care. The APN should always keep the patient in mind, know the patient's preferences before caring for him or her, and understand the patient's limits. In addition, the APN must have a solid foundation for evidence-based practice to run the correct tests. Finally, a crucial step for an APN when striving to improve practice should be reflection. On a daily basis, healthcare providers have made mistakes. The APN should use these mistakes to educate himself or herself as well as the entire healthcare team to grow and finesse clinical practice skills.

The systematic process of conducting a differential diagnosis and using the APN's critical thinking skills guided clinical decision making. By employing critical thinking skills and accruing a knowledge base from experience, the APN can deduce an illness from a list of diagnoses, starting with the most critical diagnosis and eliminating other possible diagnoses depending on the patient's pertinent signs and symptoms. A thorough and accurate assessment should be the 
first step when conducting a differential diagnosis to enable the APN to differentiate potential diagnoses with an end goal of diagnosing the patient properly and enabling appropriate healing to occur.

Coutts [2] provided a clear example of a case early in her APN career that ended in a detrimental outcome due to clinical oversight. A patient presented with a severe headache that penetrated mainly behind the right eye along with vomiting. The patient had an elevated blood pressure, and at the patient's request, Coutts treated the patient's severe headache at the beginning of the visit. Coutts consulted the physician who indicated an inability to admit all patients who had a headache. Based on the doctor's response, Coutts diagnosed the patient with a migraine and encouraged the patient to return the next day.

The next day, the woman was unconscious and presented with left-sided weakness. Because Coutts [2] did not conduct a complete neurological history and examination on the patient, she missed red flag symptoms such as meningitis, subarachnoid hemorrhage, and cerebral lesions. When linking differential diagnosis to the APN, the APN must utilize the five domains of advanced practice, including "autonomy, critical thinking, decision making, values-based care and improving practice". In the case study, Coutts disregarded her autonomy. Although she was the practitioner who physically assessed the patient, Coutts allowed the opinion of the doctor to overrule her own.
Her lack of values-based care prevented Coutts from conducting a thorough exam, causing her to miss key information that harmed the patient. Coutts misdiagnosed the patient due to the lack of a proper assessment. As a new APN, the subsequent debrief of the case ultimately improved her future role as an APN because the reconsideration of the case taught her never to forget the five domains of advanced practice.

Researching the concept differentiate in consideration of the influence of Dewey's [4] philosophy of hands-on learning answered important nursing practice questions. In the healthcare field, the APN was able to gain more insight into signs and symptoms to develop better treatment plans for various disease states through hands-on learning rather than solely textbook learning. Clinical instruction, simulation experiences, and preceptorship enriched the APN's educational experience due to the authenticity of the training; however, once the student APN has earned his or her degree, learning should not be discontinued. Education should persist as time and experience have continued to accrue in the specialized healthcare field.The expansion of an APN's knowledge base continuously grew and changed as he or she stayed up-to-date on research findings and discussed the implications of the research information with colleagues. As a result, hands-on experience in tandem with knowledge of current evidencebased research assisted the APN in conducting a proper differential diagnosis.

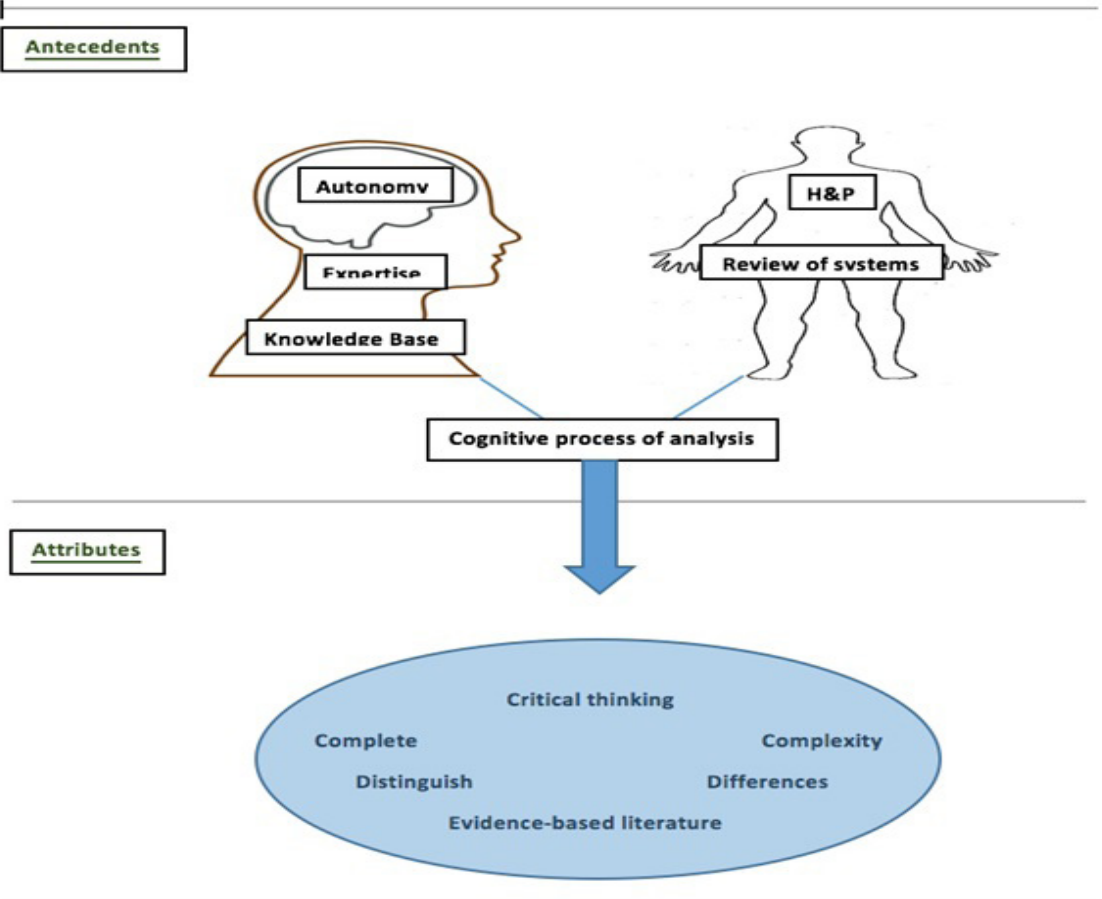

\section{Consequences}
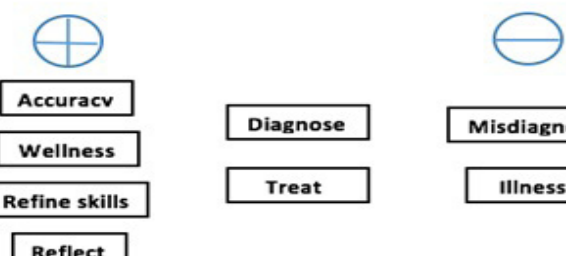

Misdiagnose

IIIness

Treat

Figure 1: Model of the differentiate concept in terms of differential diagnosis. 


\section{Discussion}

The model presented in Figure 1contains a visual of the concept analysis of differentiate applied to the APN. Included in the model are the attributes, the antecedents, and the positive and negative consequences of the concept differentiate. The concept analysis of differentiate as it applied to the APN's aim is to assist the APN in narrowing down the potential diagnoses of the patient by understanding the defining characteristics of the concept differentiate. As a result, the APN can conduct a thorough assessment of the patient, inquire about the patient's history, order the proper laboratory and other tests, delineate potential diagnoses, and ultimately determine a proper diagnosis. A sound understanding of the differential diagnosis process enables an APN to conduct a proper differential diagnosis promptly, prevent a misdiagnosis, and enhance patient safety, satisfaction, and recovery.

\section{Competing Interests}

The authors, Bonneau and Neurohr declare that they have no competing interests.

Dr. Van Sell declares she is co-author of the e-book, The Evolving Essence of theScience of Nursing: The Complexity Integration Nursing Theory.

\section{Funding}

This article was published with support from Texas Woman'sUniversity Libraries' Open Access Fund.

\section{References}

1. Walker LO, Avant KC (2011) Strategies for Theory Construction in Nursing (5th edition). Upper Saddle River, NJ: Pearson Prentice Hall.

2. Coutts B (2014) The Complex Decision Making Needed in Significant Event Analysis. Primary Health Care 24: 26-30.

3. Brown H \& Kelso L (2014) Abdominal Pain: An approach to a challenging diagnosis. AACN Adv Crit Care25: 266-278.

4. Dewey J (1897) My Pedagogic Creed. School Journal 54: 77-80.

5. Scordo K (2014) Differential Diagnosis: Correctly putting the pieces of the puzzle together. AACN Adv Crit Care 25: 230-236.

6. Phua DH, Tan NC (2013) Cognitive Aspect of Diagnostic Errors. Ann Acad Med Singapore 42: 33-41.

7. Meleis Al (2012) Theoretical Nursing: Development and Progress (4th edition). Philadelphia, PA: Lippincott Williams and Wilkins.

8. Differentiate (2016) In Merriam-Webster online dictionary.

9. Carter SL, Attel S (2013) The Diagnosis and Management of Patients with Lactose-Intolerance. Nurse Pract 38: 23-28.

10. Grimes RM, Hardwicke RL, Grimes DE, DeGarmo DS (2016) When to Consider Acute HIV Infection in the Differential Diagnosis. Nurse Pract 41: 1-5.

11. Myers T, Fecske E (2016) The "Staring" Child. Nurse Practitioner 41: 9-11.

12. Spencer P, Krieger B (2013) The Differentiation of Chronic Obstructive Pulmonary Disease from Asthma: A review of current diagnostic and treatment recommendations. Open Nurs J 7: 29-34.

13. Yeager S, Miller C (2014) Acute Nontraumatic Weakness: Overview of central nervous system differential diagnosis. AACN Adv Crit Care 25: 251265.

14. Lang GM, Beach NL, Patrician PA, \& Martin C (2013) A Cross-Sectional Study Examining Factors Related to Critical Thinking in Nursing. J Nurses Prof Dev 29: 8-15.

15. Boström A, Rudman A, Ehrenberg A, Gustavsson JP, Wallin L, et al. (2013) Factors Associated with Evidence-Based Practice Among Registered Nurses in Sweden: A national cross-sectional study. BMC Health Serv Res 13: 165 .

Int J Nurs Clin Pract 